\title{
Formation of laterally ordered quantum dot molecules by in-situ nanosecond laser interference
}

\author{
Yun-Ran Wang ${ }^{1}$, Im Sik Han ${ }^{1}$, Chao-Yuan Jin ${ }^{1,2}$ and Mark Hopkinson ${ }^{1, a)}$ \\ ${ }^{1}$ Department of Electronic and Electrical Engineering, University of Sheffield, Sheffield S3 7HQ, UK \\ ${ }^{2}$ College of Information Science and Electronic Engineering, Zhejiang University, Hangzhou 310007, China
}

a) E-mail: m.hopkinson@sheffield.ac.uk

\begin{abstract}
We demonstrate the growth and surface characterization of laterally ordered arrays of InAs quantum dot molecules (QDMs) on GaAs (100) substrates produced by a combination of in-situ interferometric nanopatterning and molecular beam epitaxy growth. Four-beam ultraviolet laser interference is applied during the growth process resulting in the formation of quasi two-dimensional islands due to localised surface diffusion. With further InAs deposition, the edges of the islands are observed to act as preferential sites for the nucleation of InAs quantum dots. Well-ordered square arrays of lateral QDMs with a period of $300 \mathrm{~nm}$ and site occupancy ranging from single dot up to hexa-molecules are obtained by varying the InAs coverage from 1.55 ML to 1.75 ML.
\end{abstract}

Semiconductor quantum dots (QDs) have become increasingly important vehicles for the development of future quantum technologies. Quantum dot molecules (QDMs), consisting of at least two vertically or laterally coupled quantum dots, ${ }^{1-3}$ have emerged as possible building blocks for novel spintronic devices, quantum cryptography and quantum computing owing to their ability of manipulating and measuring spins. ${ }^{4-7}$ Here, the electronic coupling within a QDM may enable the implementation of quantum gates by external optical or electrical manipulation of excitonic states. ${ }^{8-10}$ In recent years, vertically stacked QDMs have been extensively studied $^{2,4,10-12}$ and well-established epitaxial growth protocols have been developed. Much less attention has been paid to lateral QDMs ${ }^{13-15}$ since these require inplane patterning approaches and exacting growth techniques. Compared to vertical QDMs, where coupling is limited only to a single vertical direction, the lateral configuration provides a more flexible geometry for the manipulation of coupling as well as the potential for upscaling to a large number of quantum gates arrayed across a substrate. It could potentially allow individual QDs to be deterministically addressed whilst maintaining independent electrical or optical measurement of coherent neighbour interactions. Analogous to the behaviour of real molecular structures, the electronic properties of individual QDs can also be fine-tuned through the interactions with near neighbours. For example, it has been suggested that the fine structure splitting of the biexciton transition may be reduced or eliminated in QDMs. ${ }^{13}$

Self-assembled QDs grown via the StranskiKrastanow (S-K) growth mode exhibit high crystal quality, yet their stochastic positioning presents an obstacle for the fabrication of laterally QDMs.
Strategies have been applied to random QD arrays such as the use of $\mathrm{AsBr}_{3}{ }^{14,16,17}$ or droplet etching ${ }^{18-21}$ in order to create sites for the subsequent growth of QDMs. However, precise ordering into well-defined arrays is essential for size control and highly desirable for device integration. This requires a lithographically defined template for the epitaxial growth. Periodic single and bimolecular InGaAs QD arrays have been reported by pre-patterning the substrate surface with holes prior to growth via ex-situ electron beam lithography and etching ${ }^{14,22}$. However, such schemes are relatively low throughput and prone to introduce defects on the atomic scale due to the heterogeneous processing. An in-situ lithographic approach compatible with epitaxial growth would be an attractive alternative. In recent years, laser interference lithography has emerged as a potential method to directly fabricate ordered semiconductor nanostructures, including QD arrays. ${ }^{23-25}$ However, the formation of dense arrays of lateral QDMs assisted by laser interference has not yet been reported.

In this letter, we demonstrate an in-situ technique combining nanosecond pulsed interferometric lithography with molecular beam epitaxy (MBE) for the fast and high-efficient production of InAs/GaAs (100) QDM arrays. The formation process has been systematically investigated using atomic force microscopy (AFM). We observe, using four-beam interference, that we are able to create a regular array of island nucleation sites for QD growth and that furthermore we can manipulate the number of QDs per site to create QDMs of order from one to six by tailoring the InAs coverage. Square arrays of self-assembled QD multi-molecules with a pitch of $300 \mathrm{~nm}$ can be routinely achieved using this method. 

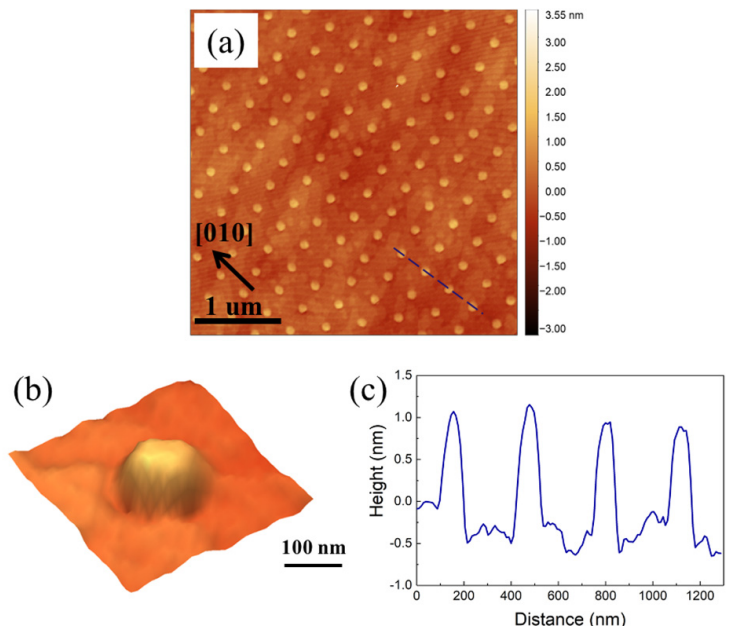

FIG. 1. (Color online) (a) AFM image with an array of quasi-2D islands induced by laser interference nanopatterning. (b) 3D AFM image of a single island. (c) Corresponding line profile of four islands along the [010] direction as marked by the dashed line in (a).

All the samples were grown on 2-inch epi-ready GaAs (100) substrates by MBE. The MBE chamber is equipped with symmetric optical viewports that allow four laser beams to converge on the growing wafer. A $355 \mathrm{~nm}$ p-polarized laser (Innolas Spitlight) operating on the third harmonic of an amplified Nd:YAG source with a pulse width of $7 \mathrm{~ns}$, a Gaussian beam diameter of $5 \mathrm{~mm}$ and a pulse energy of $40 \mathrm{~mJ}$ was employed. The four coherent beams are recombined at the centre of the substrate at an incidence angle of $58^{\circ}$. These laser parameters produce an interference pattern with a pitch of $300 \mathrm{~nm}$, with the pitch size set by the wavelength of the laser and the angle of incidence. The overlapping area of four beams on the wafer is approximately $3 \times 3$ $\mathrm{mm}$. After oxide desorption and the growth of a $500 \mathrm{~nm}$ GaAs buffer layer at a substrate temperature of $600^{\circ} \mathrm{C}$, the substrate was cooled to $500^{\circ} \mathrm{C}$ for InAs QD growth. After the deposition of 1.0 monolayer (ML) InAs at a growth rate of $0.079 \mathrm{ML} / \mathrm{s}$, single-pulse laser interference nanopatterning was performed, followed by the further deposition of an additional $0.55-0.75 \mathrm{ML}$ InAs. After 10 seconds of interruption under $\mathrm{As}_{2}$ flux, the samples were then quenched and surface characterization was then carried out by AFM in air.

Figure 1(a) shows an AFM image of a sample surface following the initial 1 ML InAs coverage and singlepulse four-beam laser interference nanopatterning. The surface shows a square array of quasi-two-dimensional islands with a period of $300 \mathrm{~nm}$ induced by the interference pattern. A three-dimensional AFM image of single island can be seen in Fig. 1(b) and the corresponding line profile of islands along the [010] crystallographic direction is presented in Fig. 1(c). The islands are typically $\sim 100 \mathrm{~nm}$ in width and $\sim 1 \mathrm{~nm}$ in height. We explain the formation process of these islands as follows. Four-beam interference leads to square pattern of interference maxima and minima on the surface. Due to the absorption of the intense ultraviolet pulse, localised heating occurs on the surface. As a result, we believe indium adatoms are capable of migrating along the surface from the interference maxima regions to the interference minima regions, driven by the thermal gradients. ${ }^{23,26}$ Consequently, the surface morphology of the initial $1 \mathrm{ML}$ InAs, which otherwise would grow as single monolayer platelets, turns into a square array of small quasi-2D islands with a period corresponding to the pattern pitch size. The height of these islands seems remarkably consistent at around $1 \mathrm{~nm}(2-3 \mathrm{ML})$ over a wide range of pulse

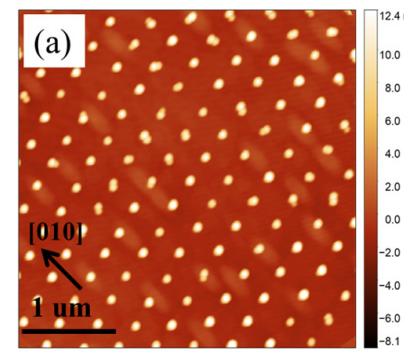

(d)

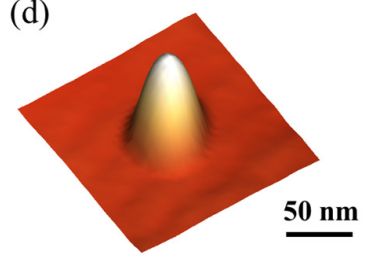

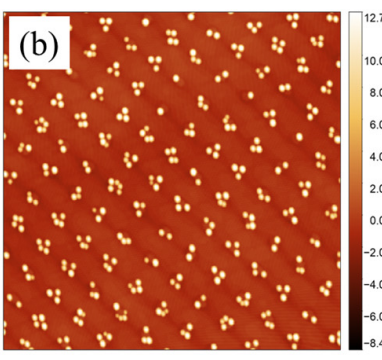

(e)

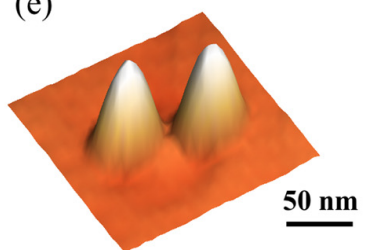

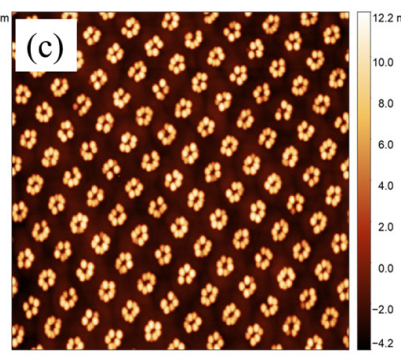

(f)

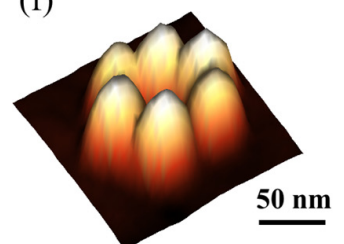

FIG. 2. (Color online) AFM surface morphologies of additional deposition of (a) $0.55 \mathrm{ML}$, (b) $0.65 \mathrm{ML}$ and (c) 0.75 ML InAs on the quasi-2D island templated surfaces in Fig. 1(a). (d-f) Corresponding 3D AFM images of enlarged single features. 
energy and is probably limited in height by the build-up of strain. However, the island width has a strong dependence on the laser energy which we believe is due to the number of diffused atoms driven by the photothermal gradient.

Overgrowth of the nanoislands with further InAs deposition results in a strain driven $\mathrm{S}-\mathrm{K}$ transition and the formation of QDs and QDMs. Figures 2(a)-(c) illustrate the surface evolution during the growth of InAs QDs/QDMs with incremental InAs amounts from $0.55 \mathrm{ML}$ to $0.75 \mathrm{ML}$ onto a laser-structured surface prepared identically to Fig. 1(a). With a further $0.55 \mathrm{ML}$ InAs deposition, we observe the formation of an imperfect single QD array with the original quasi-2D islands invisible (Fig. 2(a)). The height of these single QDs is in the range 12-15 nm. Fig. 2(b) shows the surface morphology with $0.65 \mathrm{ML}$ of InAs deposited on the island-templated surface. This shows clearly that QDs are formed at the island edges. There is a fluctuation in QD size and occupancy in this sample, indicating that the InAs amount is not optimum for the particular island geometry. However, some equal size bi-QDMs can be seen, as shown in Fig. 2(e). A typical bi-QDM in this sample consists of two dots with identical QD widths and heights of $\sim 50 \mathrm{~nm}$ and $\sim 15 \mathrm{~nm}$, respectively and with a separation between the two dots equivalent to the original island size $(\sim 100 \mathrm{~nm})$. With an InAs coverage of 1.75 ML, as shown in Fig. 2(c), more indium atoms can accumulate, thereby forming a greater density of QDs around the islands. A typical symmetric hexa-QDM consisting of six QDs in a radial molecule is shown in Fig. 2(f). The interdot distance of
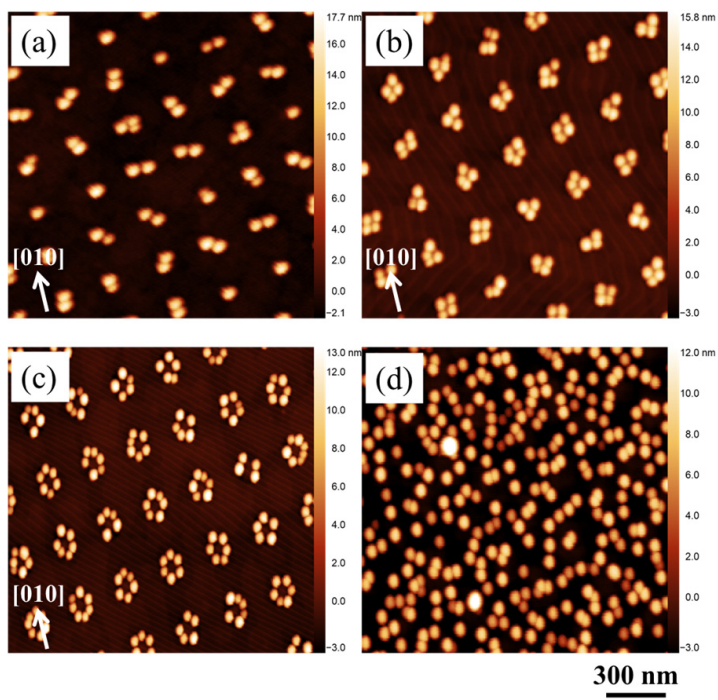

FIG. 3. $2 \times 2 \mu \mathrm{m}^{2}$ AFM images of sample surfaces with various InAs coverage (a) $1.6 \mathrm{ML}$, (b) $1.7 \mathrm{ML}$, and (c) 1.75 ML. (d) Non-irradiated surface region with 1.75 ML InAs coverage. this molecule is greatly reduced, which makes strong coupling possible. ${ }^{27}$ The individual QDs in this case are generally $40-50 \mathrm{~nm}$ in width and $12-15 \mathrm{~nm}$ in height. Note in all cases the original quasi-2D islands are no longer present and therefore it is assumed that the growth of QDs is at the expense of material coming from these islands. The formation of quasi-2D islands and their role in QD nucleation is a unique observation in our work. This is in contrast to typical methods relying on an array of etched holes. ${ }^{15}$ The islands act as preferential nucleation sites for InAs QDMs, since QDs tend to nucleate at step edge locations. ${ }^{28,29}$

By depositing slightly different amounts of InAs onto the initial $1 \mathrm{ML}$ InAs island-templated surface, the number of QDs per island site varies accordingly. Figures 3(a)-3(c) demonstrate the resulting surface morphologies for different InAs coverages of 1.6 ML, 1.7 ML and 1.75 ML, respectively. For comparison, Fig. 3(d) presents a non-patterned sample of freestanding QDs with an InAs coverage of 1.75 ML which shows a high QD density of $\sim 7 \times 10^{9} \mathrm{~cm}^{-2}$. The statistical analysis for these samples, including the QD occupancy in the molecule and the QD height distributions are shown in Figure 4. In Fig. 4(a), the InAs coverage which is close to the critical thickness for 2D to $3 \mathrm{D}$ transition ( 1.6 ML) leads to the nucleation of one to three QDs per island site, of which $\sim 75 \%$ are bi-molecules and $20 \%$ are single dots. This shows a substantial increase in height and narrower distribution that the un-patterned case. With increased InAs coverage of 1.7 ML (see Fig. 3(b) and Fig. 4(b)), tri-molecules and quad-molecules are formed also showing good size uniformity. The average height of these QDMs is around $15 \mathrm{~nm}$. When the InAs amount is supplied to $1.75 \mathrm{ML}$ as shown in Fig. 3(c) and Fig. 4(c), we observe the formation of pentaand hexa-molecules with an average height of $\sim 12 \mathrm{~nm}$, and a QD density of $\sim 4 \times 10^{9} \mathrm{~cm}^{-2}$. In Fig. 4(d) the unpatterned QDs have a relatively broader size distribution centred at $\sim 8.5 \mathrm{~nm}$ and with a few large clusters formed by coalescence. These results are typical of what we would expect for random QDs using this (non-optimum) InAs deposition amount. All the patterned InAs QDs grown have larger sizes and exhibit better homogeneity compared to the un-patterned QDs. Note, the growth of interstitial QDs on the planar areas between island sites is almost completely suppressed. This indicates that the formation of the quasi-2D islands by the direct application of laser interference is extremely efficient at inducing lateral patterning.

At present we can only reproducibly pattern an area $\sim 100 \mu \mathrm{m}^{2}$ due to beam profile inhomogeneity, chiefly arising from the superposition of four Gaussian beam intensity profiles. Such profiles are somewhat inevitable given the long $(\sim 3 \mathrm{~m})$ distance between the laser and 


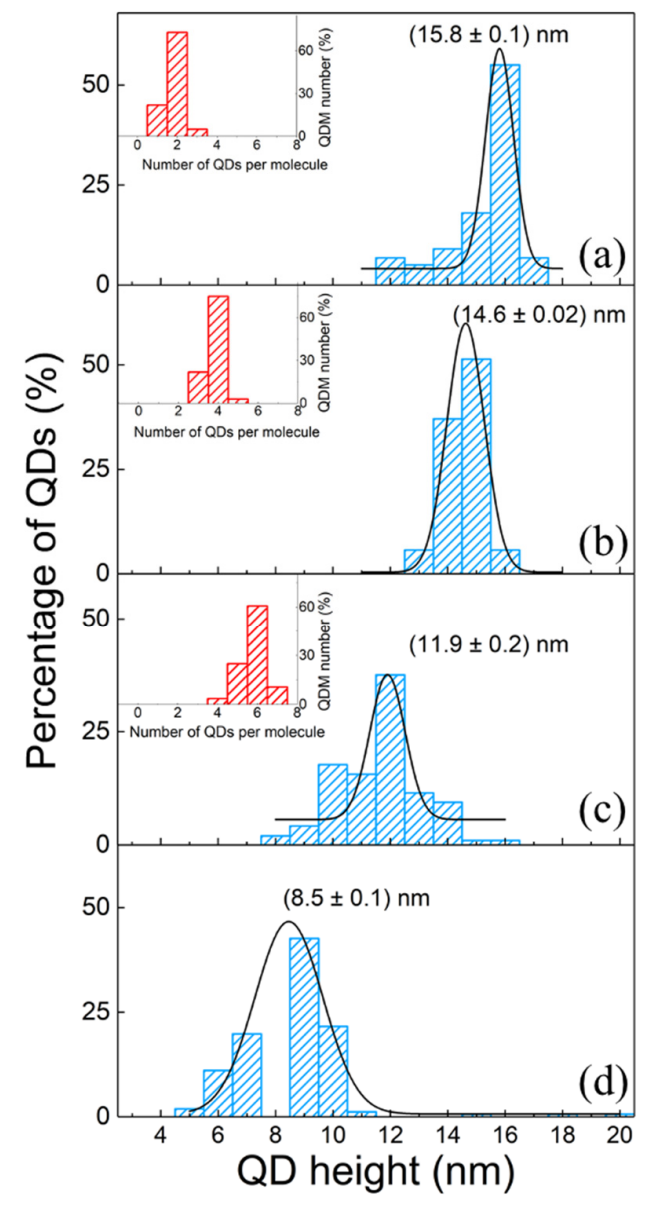

FIG. 4. Histograms of InAs QD height distribution for laser-assisted grown QDs with InAs coverage of (a) 1.6 ML, (b) $1.7 \mathrm{ML}$, and (c) 1.75 ML. (d) Non-irradiated QDs with 1.75 ML InAs coverage. The insets show the corresponding QD occupancy per molecule.

substrate. We are working on improving this with the use of additional optical components. However, it is worthwhile to point out that we are patterning such an area with a single $7 \mathrm{~ns}$ pulse and this represents a very high throughput compared to other lithographic techniques. The method could always be integrated within a step and repeat process to achieve larger areas. Finally, we are able to report that we observe strong narrow photoluminescence emissions in the range of 1.2-1.25 $\mu \mathrm{m}$ at room temperature from these QDM ensembles. Further studies are underway to evaluate their detailed optical properties.

In conclusion, dense square arrays of lateral InAs quantum dot molecules (QDMs) have been obtained by a combination of in-situ single-pulse laser interference nanopatterning and MBE growth. Quasi-twodimensional islands are formed on a sub-critical 1 monolayer InAs surface by diffusion under the influence on an intense localised thermal gradient. QDMs of varying number of dots are nucleated at the edges of these nanoislands, with the yield controlled by the amount of InAs coverage. The resulting QD/QDMs range from single dots, bi-, quad-, up to hexamolecules. As the number of dots per molecule increases, there is an increase in the QD height and an improved size uniformity. This technique offers a powerful way to fabricate precisely ordered lateral QDMs for the study of coupled QD systems and the potential realization of coupling-based quantum information devices.

The authors gratefully acknowledge the support from the EU H2020 program 'Nanostencil' and EPSRC.

The data that support the findings of this study are available from the corresponding author upon reasonable request.

\section{REFERENCES}

${ }^{1}$ L. Wang, A. Rastelli, S. Kiravittaya, M. Benyoucef, O. G. Schmidt, Adv. Mater. 21(25-26), 2601-2618 (2009).

${ }^{2}$ M. L. Kerfoot, A. O. Govorov, C. Czarnocki, D. Lu, Y. N. Gad, A. S. Bracker, M. Scheibner, Nat. Commun. 5(1), 1-6 (2014).

${ }^{3}$ J. Peng, C. Hermannstädter, M. Witzany, M. Heldmaier, L. Wang, S. Kiravittaya, G. Bester, Phys. Rev. B 81(20), 205315 (2010).

${ }^{4}$ M. Khoshnegar, T. Huber, A. Predojević, D. Dalacu, M. Prilmüller, J. Lapointe, G. Weihs, Nat. Commun. 8(1), 18 (2017).

${ }^{5}$ M. Bayer, P. Hawrylak, K. Hinzer, S. Fafard, M. Korkusinski, Z. R. Wasilewski, A. Forchel, Science 291(5503), 451-453 (2001).

${ }^{6}$ D. Loss, D. P. DiVincenzo, Phys. Rev. A 57(1), 120 (1998).

${ }^{7}$ E. A. Stinaff, M. Scheibner, A. S. Bracker, I. V. Ponomarev, V. L. Korenev, M. E. Ware, D. Gammon, Science 311(5761), 636-639 (2006).

${ }^{8}$ G. Burkard, G. Seelig, D. Loss, Phys. Rev. B 62(4), 2581 (2000).

${ }^{9}$ L. Robledo, J. Elzerman, G. Jundt, M. Atatüre, A. Högele, S. Fält, A. Imamoglu, Science 320(5877), $772-775$ (2008). ${ }^{10}$ C. Emary, L. J. Sham, Phys. Rev. B 75(12), 125317 (2007).

${ }^{11}$ H. J. Krenner, M. Sabathil, E. C. Clark, A. Kress, D. Schuh, M. Bichler, J. J. Finley, Phys. Rev. Lett. 94(5), 057402 (2005).

${ }^{12}$ A. S. Bracker, M. Scheibner, M. F. Doty, E. A. Stinaff, I. V. Ponomarev, J. C. Kim, D. Gammon, Appl. Phys. Lett. 89(23), 233110 (2006).

${ }^{13}$ S. Fillipov, Y. Puttisong, Y. Huang, I. A. Buyanova, S. Suraprapapich, C. W. Tu, W. M. Chen, ACS nano 9(6), 5741-5749 (2015). 
${ }^{14}$ L. Wang, A. Rastelli, S. Kiravittaya, P. Atkinson, F. Ding, C. B. Bufon, O. G. Schmidt, New J. of Phys. 10(4), 045010 (2008).

${ }^{15}$ R. Songmuang, S. Kiravittaya, O. G. Schmidt, Appl. Phys. Lett. 82(17), 2892-2894 (2003).

${ }^{16}$ S. Kiravittaya, R. Songmuang, N. Y. Jin-Phillipp, S. Panyakeow, O. G. Schmidt, J. Cryst. Growth 251(1-4), 258-263 (2003).

${ }^{17}$ E. Zallo, P. Atkinson, L. Wang, A. Rastelli, O. G. Schmidt, Phys. status solidi (b) 249(4), 702-709 (2012).

${ }^{18}$ J. H. Lee, Z. M. Wang, N. W. Strom, Y. I. Mazur, G. J. Salamo, Appl. Phys. Lett. 89(20), 202101 (2006).

${ }^{19}$ B. L. Liang, Z. M. Wang, X. Y. Wang, J. H. Lee, Y. I. Mazur, C. K. Shih, G, J, Salamo, ACS nano 2(11), 22192224 (2008).

${ }^{20}$ M. Yamagiwa, T. Mano, T. Kuroda, T. Tateno, K. Sakoda, G. Kido, F. Minami, Appl. Phys. Lett. 89(11), 113115 (2006).

${ }^{21}$ H. Kim, J. S. Kim, Phys. status solidi (b) 256(6), 1800532 (2019).

${ }^{22}$ H. Heidemeyer, C. Müller, O. G. Schmidt, J. Cryst. Growth 261(4), 444-449 (2004).

${ }^{23}$ C. M. Clegg, H. Yang, Solar Energy Materials and Solar Cells 108, 252-255 (2013).

${ }^{24}$ W. Zhang, Z. Shi, D. Huo, X. Guo, F. Zhang, L. Chen, C. Peng, Appl. Phys. Lett. 112(15), 153108 (2018).

${ }^{25}$ Y. R. Wang, M. Hopkinson, I. S. Han, S. Behera, C. Y. Jin, Nanostructures, and Quantum Materials: Growth, Characterization, and Modeling XVII. International Society for Optics and Photonics 11291, 1129107 (2020). ${ }^{26}$ Y. R. Wang, C. Y. Jin, C. H. Ho, S. Chen, H. Francis, M. Hopkinson, IET Opto. 13(1), 7-11 (2018).

${ }^{27}$ R. Parvizi, Phys. B: Condensed Matter 482, 51-57 (2016).

${ }^{28}$ E. Placidi, F. Arciprete, V. Sessi, M. Fanfoni, F. Patella, A. Balzarotti, Appl. Phys. Lett. 86(24), 241913 (2005)

${ }^{29}$ F. Arciprete, E. Placidi, R. Magri, D. DelGaudio, F. Patella, J. of Mater Res. 28(23), 3201-3209 (2013). 